\title{
Homeostatic regulation of lipid droplet content in mammalian oocytes and embryos
}

\author{
Megumi Ibayashi¹, Ryutaro Aizawa¹, Junichiro Mitsui ${ }^{1,2}$ and Satoshi Tsukamoto®1 \\ ${ }^{1}$ Laboratory Animal and Genome Sciences Section, National Institutes for Quantum Science and Technology, \\ Anagawa, Chiba, Japan and ${ }^{2}$ Department of Comprehensive Reproductive Medicine, Graduate School, Tokyo \\ Medical and Dental University, Tokyo, Japan
}

Correspondence should be addressed to S Tsukamoto; Email: tsukamoto.satoshi@qst.go.jp

\begin{abstract}
Lipid droplets (LDs) consist of a core of neutral lipids such as triacylglycerols and cholesteryl esters covered by a phospholipid monolayer. Recent studies have shown that LDs not only store neutral lipids but are also associated with various physiological functions. LDs are found in most eukaryotic cells and vary in size and quantity. It has long been known that mammalian oocytes contain LDs. Porcine and bovine oocytes contain substantial amounts of LDs, which cause their cytoplasm to darken, whereas mouse and human oocytes are translucent due to their low LD content. A sufficient amount of LDs in mammalian oocytes has been thought to be associated with oocyte maturation and early embryonic development, but the necessity of LDs has been questioned because embryonic development proceeds normally even when LDs are removed. However, recent studies have revealed that LDs play a crucial role during implantation and that maintaining an appropriate amount of LDs is important for early embryonic development, even in mammalian species with low amounts of LDs in their oocytes. This suggests that a fine-tuned balance of LD content is essential for successful mammalian embryonic development. In this review, we discuss the physiological importance of LDs in mammalian oocytes and preimplantation embryos based on recent findings on LD biology.

Reproduction (2021) 162 R99-R109
\end{abstract}

\section{Introduction}

Most researchers who have studied mammalian oocytes and fertilized embryos under a microscope have surely wondered why they appear semi-transparent, dark, or speckled. The cause of this variation in appearance is the lipid droplet (LD) content of the oocyte and embryo. LDs consist of a core of neutral lipids such as triacylglycerols (TG) and cholesteryl esters surrounded by a phospholipid monolayer (Tauchi-Sato et al. 2002). They are universally present in eukaryotic cells, from yeast to humans. Various proteins located on the LD surface regulate the size and quantity of the LD as well as the intracellular distribution of the LDs by synthesizing neutral lipids and protecting the LDs themselves. A core set of structural proteins, such as those in the perilipin family (PLIN1-5), has been found to decorate LDs in mammals, although their tissue distribution varies (discussed in the next section). Additionally, recent proteomics studies have revealed that a wide variety of proteins are expressed on the LD surface. Some of these proteins are not involved in lipid metabolism, leading LDs to be recognized as organelles with various functions beyond mere lipid storage. For example, LDs play a pivotal role in fatty acid trafficking, gene expression, protein storage, viral infection, host defense, and neuronal homeostasis ( $\mathrm{Li}$ et al. 2012,
Rambold et al. 2015, loannou et al. 2019, Laufman et al. 2019, Bosch et al. 2020, Mejhert et al. 2020).

Because LDs can be easily seen under a microscope, their presence in mammalian eggs has long been known. There is considerable interspecies variation in the LD content of mammalian oocytes and early embryos. For example, the cytoplasm of porcine and bovine oocytes/ embryos appears dark due to their high LD content, whereas the cytoplasm of mouse and human oocytes/ embryos appears clear because of their low LD content (for a more detailed discussion of the variation in lipid content among mammalian species, see Bradley \& Swann 2019). LDs are not considered essential for mammalian embryonic development, as a result of the pioneering work of Nagashima et al. (1995) who showed that porcine embryos develop normally even when LDs are removed. However, recent studies have provided new insights into the role of LDs in mammalian embryos, especially in relation to embryonic diapause (ED) (Arena et al. 2021) and the regulation of LD content (Aizawa et al. 2019) (discussed in the following section on the role of LDs in ED and the homeostatic regulation of LD content in oocytes and early embryos, respectively). It appears that LD synthesis (lipogenesis) and LD degradation (lipolysis/lipophagy; see also Box 1) in mammalian oocytes/embryos are homeostatically 


\section{Box 1. Lipophagy}

Lipophagy is the selective degradation of LDs by autophagy - a cellular degradation system in which cytoplasmic components sequestered by a double membrane structure called an autophagosome are degraded by lysosomes (Mizushima 2007). Autophagy degrades cytoplasmic components either randomly (non-selectively) or by targeting specific organelles and proteins. The latter is referred to as selective autophagy. Given that autophagosomes are not equipped to distinguish a particular target, binding molecules called autophagy adapters are required to target organelles and proteins. The molecules that bind LDs to autophagosomes and the mechanism by which lipophagy is induced remain to be elucidated. However, the expression of the autophagy adapter protein p62/Sqstm1 (Ichimura et al. 2008) on the LD surface in cultured mammalian cells or early mouse embryos results in the semi-forced degradation of some LDs via the activation of intrinsic autophagy (Tatsumi et al. 2018). This method is called forced lipophagy. Moreover, it was recently revealed that LD quantity and size are coordinately regulated by lipolysis and lipophagy (Schott et al. 2019).

regulated to maintain appropriate amounts of LDs, regardless of interspecies differences.

In general, LDs are broken down as needed, and the resulting fatty acids (metabolites) are utilized as an energy source in the cell. In mammals, oocyte maturation and early embryonic development are considered to be energy-consuming processes. Therefore, it is reasonable to store intracellular LDs with high ATP production efficiency in preparation for increased energy demands, even in mouse oocytes and early embryos with relatively low LD content (for a more in-depth review of the necessity of lipid metabolism for oocyte maturation and early embryonic development, see Sturmey et al. 2009, McKeegan \& Sturmey 2011, Dunning et al. 2014). However, because mature oocytes and early cleavage-stage embryos develop in nutrientrich environments (i.e. follicular fluid, oviductal fluid, and culture medium), it is plausible that LDs play a role other than as an energy source in these cells. In this review, we discuss the physiological roles of LDs in mammalian oocytes/embryos based on the findings of recent research on LDs.

\section{LD synthesis and LD-associated proteins}

Given that many of the enzymes required for LD synthesis (e.g. diglyceride acyltransferase, which catalyzes the final step of TG synthesis) are present on the surface of the endoplasmic reticulum (ER) (Buhman et al. 2001), LDs are thought to bud from the cytoplasmic side of the ER bilayer and to dissociate after maturation, a process that has been discussed in detail in a previous review (Walther et al. 2017). In addition to neutral lipid synthetases, other proteins from the ER and cytoplasm are expressed sequentially on the LDs during the budding and maturation process (Kassan et al. 2013). Many such proteins involved in LD biogenesis have been identified by proteome analysis. PLIN1-5 are a representative group of such proteins (Brasaemle et al. 2004, Bickel et al. 2009). All members are present on LDs, but their tissue expression patterns are different. For example, PLIN1 and PLIN4 are expressed in adipose tissues and steroid-producing cells, PLIN2 and PLIN3 are ubiquitously expressed, and PLIN5 is highly expressed in oxidative tissues such as heart and skeletal muscle (Greenberg et al. 1993, Servetnick et al. 1995, Brasaemle et al. 1997, Yamaguchi et al. 2006). Many of the PLIN proteins expressed on the LD surface are thought to be involved in the regulation of neutral lipid quantity by preventing lipase attack (lipolysis). Indeed, the tissue LD content is greatly reduced in Plin 1 and Plin2 knockout mice, which are resistant to obesity (MartinezBotas et al. 2000, Tansey et al. 2001, Chang et al. 2006, McManaman et al. 2013). In addition, the expression of PLIN proteins changes according to the size of the LD. For example, PLIN3 and PLIN4 are expressed in smaller LDs during the early phase of formation. As the LD grows, more PLIN2 is expressed, and PLIN1 becomes predominant when the LD reaches maturity (Wolins et al. 2005).

Mammalian oocytes and early embryos express PLIN2 (Sastre et al. 2014, Zhang et al. 2014), which is localized on the LD surface (Yang et al. 2010). When GFP-PLIN2 (a fusion protein consisting of green fluorescent protein and PLIN2) is expressed in mouse embryos, it localizes on the LD surface (Fig. 1A). PLIN proteins are thought to be involved in the regulation of LD size and quantity in mammalian oocytes and early embryos, as in cultured mammalian cells. To date, about 200 proteins involved in LD functions have been identified, although some of these are unrelated to lipid metabolism (Hodges \& Wu 2010, Bersuker et al. 2018). Moreover, it is suspected that LD functions are diversified by the presence of proteins such as PLIN proteins, which are expressed according to the specific type of cell and tissue as well as the size of the LD.

\section{Interaction between LDs and other organelles}

In addition to the ER, LDs interact with nearly all other intracellular organelles, including the mitochondria, Golgi apparatus, lysosomes, and peroxisomes (Valm etal. 2017) as well as the nucleus (Romanauska \& Kohler 2018). By interacting with various organelles, LDs are thought to exchange lipids and metabolites efficiently. Moreover, the interaction of LDs with different organelles changes according to the nutritional status of the cell (Valm et al. 2017). For example, the frequency of interactions of LDs with mitochondria and lysosomes increases under starvation conditions. LDs are frequently found in close 

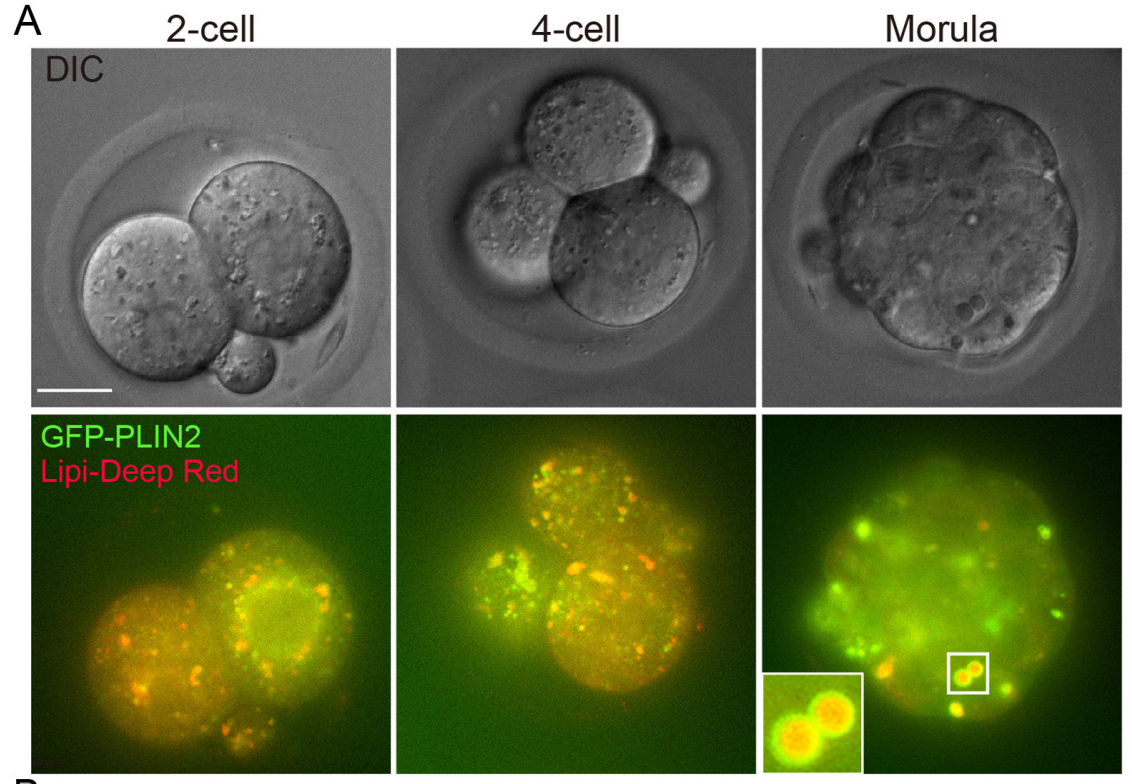

B
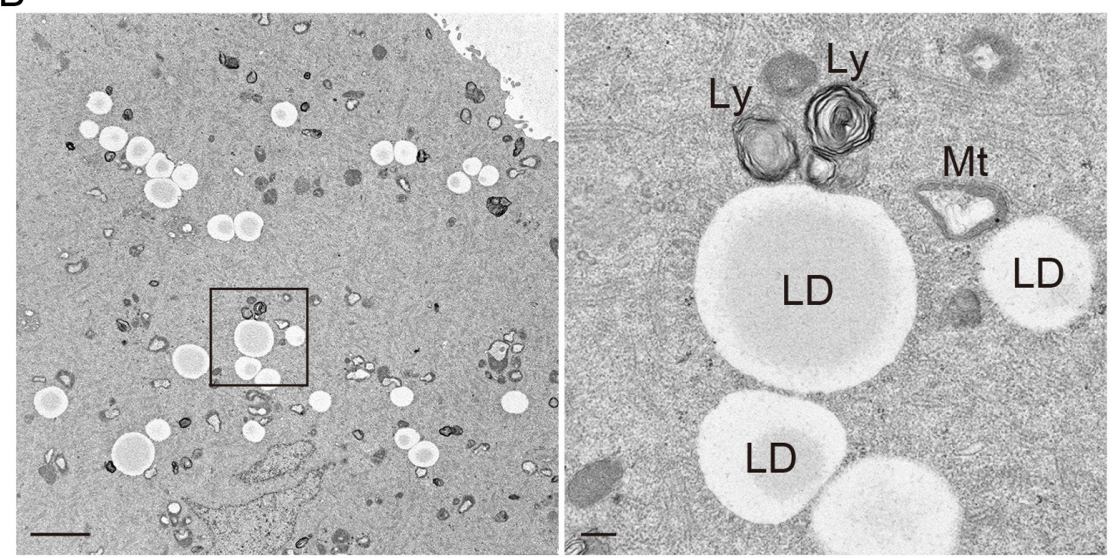

Figure 1 LDs in early mouse embryos. (A) Fluorescence microscopic images showing LDs (green, GFP-PLIN2; red, Lipi-Deep Red) in developing embryos. Fertilized one-cell embryos microinjected with mRNA encoding GFP-PLIN2 were stained with neutral lipid dye $(0.1 \mu \mathrm{mol} / \mathrm{L}$, Lipi-Deep Red, Dojindo, LD04) at the indicated embryonic stages and imaged by confocal microscopy, as described previously (Aizawa et al. 2019). Inset shows a higher magnification image of the boxed area. $\mathrm{DIC}$, differential interference contrast. Scale bar, $20 \mu \mathrm{m}$. (B) Electron micrograph showing LDs in a four-cell embryo. The right panel shows a higher magnification image of the boxed area. Mitochondria (Mt) and lysosomes (Ly) can be found in close proximity to LDs. Scale bars, $2 \mu \mathrm{m}$ (left) and $200 \mathrm{~nm}$ (right). The embryos used in this studies were collected from C57BL/6 mice. association with mitochondria and lysosomes in mouse early embryos (Fig. 1B). This close association between LDs and mitochondria is also found in porcine oocytes (Sturmey et al. 2006). These interactions are presumed to occur due to increased $\beta$-oxidation (fatty acid transport) and autophagy-mediated LD degradation (lipophagy) to compensate for a shortage of fatty acids in the cell. In contrast, LDs interact less frequently with peroxisomes and more frequently with lysosomes when there is an overabundance of fatty acids. Such interactions occur at the membrane contact site (MCS) of the organelles (Prinz 2014, Thiam \& Dugail 2019). Much remains to be elucidated about the molecular mechanism of contact between LDs and other organelles, which is a current hot topic in cell biology research.

Recently, it has been shown that LDs are formed on the inner nuclear membrane (INM), where many neutral lipid synthesis-related proteins are present (Ohsaki et al. 2016, Soltysik et al. 2021). Such LDs formed on the INM supply phospholipids during nuclear membrane extension and are involved in transcriptional regulation.
However, because most studies on mammalian oocytes and early embryos have focused on the interactions between LDs and mitochondria, there is still much to be discovered about the interaction of LDs with other organelles.

\section{Changes in the distribution and morphology of LDs}

To maintain cellular lipid and energy homeostasis, LDs are degraded by lipolysis and/or lipophagy. The resulting degradation products are fatty acids, which are taken up into the mitochondria and converted to energy (ATP) by $\beta$-oxidation (see the review by Zechner et al. 2017). Under such conditions, LDs come into close proximity with mitochondria (Herms et al. 2015, Rambold et al. 2015), probably to facilitate the efficient uptake of fatty acids into the mitochondria as well as to prevent excessive leaching of fatty acids in the cell (i.e. to prevent lipotoxicity and abnormal lipid signaling) (Rambold et al. 2015, Nguyen et al. 2017). Closer association between LDs and mitochondria 
is regulated by a protein-mediated interaction at the MCS. For example, overexpression of PLIN5 in cultured cardiomyocytes induces close apposition of mitochondria and LDs (Wang et al. 2011). PLIN5 is highly expressed in tissues with high oxidative stress, including the liver, heart, and skeletal muscle, which indicates that LDs and mitochondria come into contact in these tissues through the interaction of PLIN5 on the LD surface with mitochondrial membrane proteins (Benador et al. 2018). However, contact between LDs and mitochondria is also observed in cells and tissues with low expression of PLIN5, suggesting that interaction between LDs and mitochondria might be regulated by other molecular machinery.

Meanwhile, PLIN2 and PLIN3 are expressed in mammalian oocytes/embryos (bovine, Sastre et al. 2014; porcine, Zhang et al. 2014; mouse, Yang et al. 2010), and LDs are found near mitochondria around the cell membrane of porcine embryos (Sturmey et al. 2006). Electron microscopy has revealed that the electron density of LDs in porcine oocytes temporarily declines immediately after fertilization (Kikuchi et al. 2002). This change in LD electron density is related to the change in fatty acid composition of TG. It is possible that this change may be the result of LD catabolism (lipolysis and/or lipophagy) induced to secure the energy required for the series of events that occur after fertilization (i.e. cortical granule release, sperm nuclear decondensation, pronucleus formation, and DNA replication). The immediate post-fertilization decrease in TG is also observed in other mammals, but its physiological purpose is not known.

During lipolytic stimulation, LDs decrease in size and disperse in the cytoplasm. Despite differences among mouse strains, LDs stored in mature oocytes often form clusters of small LDs and then disperse in the cytoplasm after fertilization. (The morphological changes of LDs in mouse oocytes and early embryos will be discussed later.) LD dispersion is also observed in metaphase stage II (MII) oocytes cultured in a medium without pyruvate (to simulate carbohydrate starvation conditions) (Bradley et al. 2016). These observations suggest that LDs miniaturize and disperse into the cytoplasm as a result of catabolism by lipolysis and/or lipophagy in response to fertilization stimuli and the energy requirements of the cell. In cultured mammalian cells, starvation activates AMP-activated protein kinase (AMPK) signaling, which mediates contact between dispersed LDs and mitochondria around the cell periphery in order to accelerate fatty acid uptake into the mitochondria (i.e. promotes $\beta$-oxidation) (Herms et al. 2015). This enhanced $\beta$-oxidation is suppressed by carnitine palmitoyltransferase-I inhibitor (etomoxir), which inhibits mitochondrial uptake of fatty acids.

Culturing mammalian oocytes and early embryos with etomoxir decreases the rate of oocyte maturation and embryonic development (Downs et al. 2009,
Dunning et al. 2010, Valsangkar \& Downs 2013). In contrast, these processes are promoted when $\beta$-oxidation is activated by adding L-carnitine to the culture medium (Downs et al. 2009, Dunning \& Robker 2012). Moreover, when mouse MII oocytes are cultured with etomoxir, LDs tend to become clustered (Bradley et al. 2019), suggesting a correlation between $\beta$-oxidation activity and LD morphology. Further studies are needed to determine whether the changes in morphology and distribution of LDs in oocytes and early cleavagestage embryos according to the $\beta$-oxidation level are regulated via the same molecular mechanisms as in the cultured cells.

\section{LD migration}

As mentioned above, LDs contact various organelles in addition to the ER, but how do the LDs travel within the cell to contact other organelles? In cultured mammalian cells, the cytoskeleton (microtubules and actin filaments) is involved in LD migration. Short-distance migration occurs through Brownian motion of the LDs themselves, whereas long-distance migration is mediated through interactions with the cytoskeleton. It is likely that LDs in mammalian oocytes and early embryos migrate using similar mechanisms. Indeed, active Brownian motion of LDs isolated from mouse MII oocytes has been observed under a microscope (Video 1). Meanwhile, simultaneous treatment of mouse embryos with microtubule polymerization inhibitor (nocodazole) and actin polymerization inhibitor (cytochalasin) restricts LD migration (Watanabe et al. 2010). But how LDs interact with the cytoskeleton and how LDs destined for shortand long-distance migration are distinguished within the cell remains to be elucidated. Because LDs show remarkable variation in protein and lipid composition (Wolins et al. 2005, Herms et al. 2013, Wilfling et al. 2013), these differences in characteristics might contribute to the determination of migration distance and distribution within the cell.

Video 1 Brownian movement of LDs isolated from mouse MII oocytes. LDs isolated from MII oocytes by using a two-step centrifugation technique (see Box 2) were packed into an empty oocyte in which the oocyte contents were removed beforehand and therefore only the zona pellucida remained intact. This video (http:// movie-usa.glencoesoftware.com/video/10.1530/REP21-0238/video-1) is available from the online version of the article at https://doi.org/10.1530/REP-21-0238.

\section{The role of LDs in mammalian oocytes and early embryos}

The amount of LDs in mammalian oocytes differs greatly among species, suggesting that LDs have species-specific functions in addition to their universal 

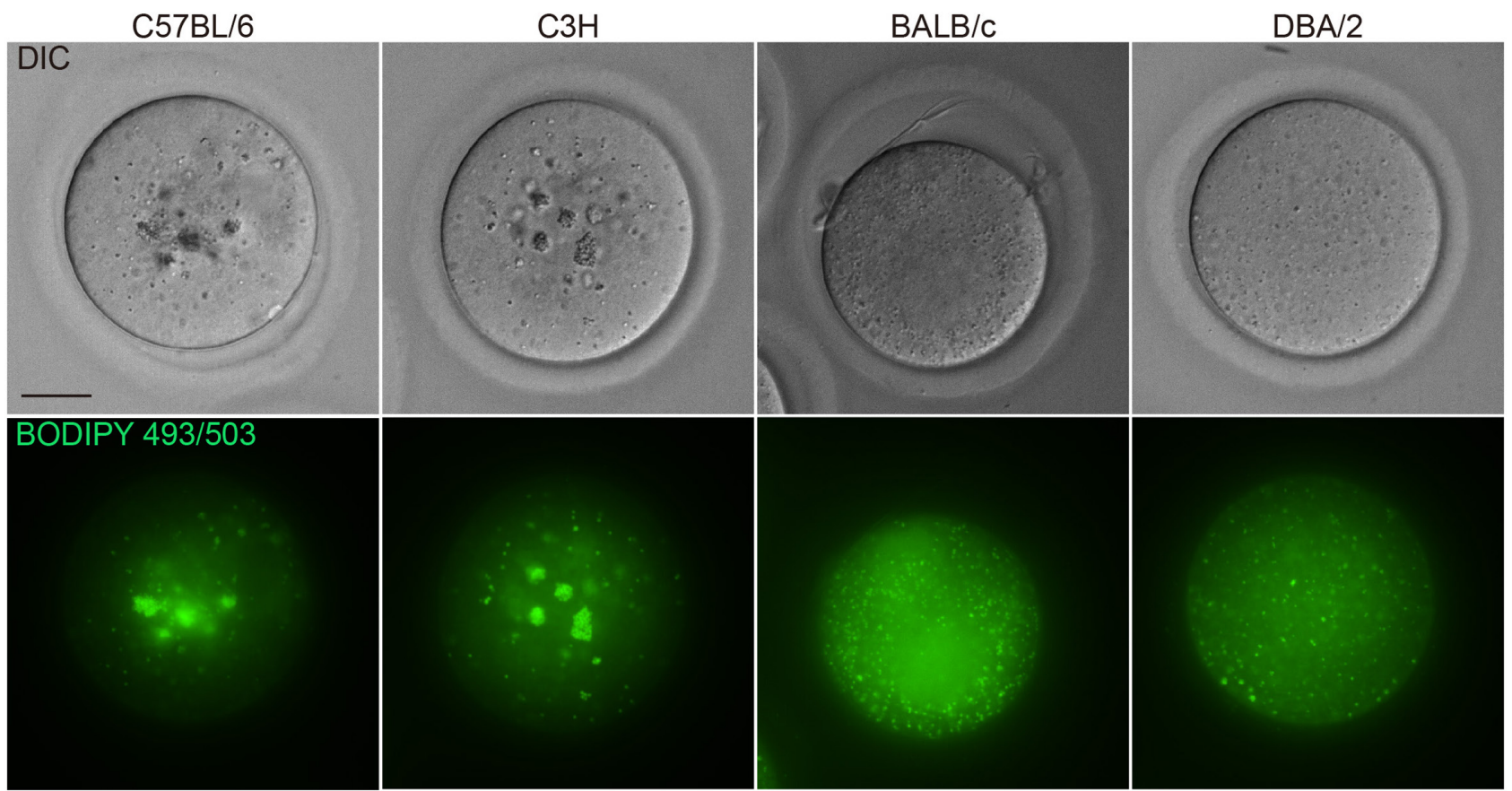

Figure 2 LD morphology of MII oocytes in different mouse strains. MII oocytes collected from the indicated female mice were stained with BODIPY 493/503 and immediately observed by confocal fluorescence microscopy, as described previously (Aizawa et al. 2019). DIC, differential interference contrast. Scale bar, $20 \mu \mathrm{m}$.

functions. Moreover, the distribution and morphology of LDs differ among mouse strains, even though the LD content of mouse oocytes is low (Fig. 2). This section focuses on LD research in mouse oocytes and early embryos and discusses the possible roles of LDs in early embryonic development as well as factors that influence LD quantity.

\section{Morphological changes in LDs after fertilization}

The LDs in mature (ovulated) oocytes and in early embryos immediately after fertilization are often small and are dispersed throughout the cytoplasm or may be partially clustered. However, larger LDs are observed after compaction (morula stage; Fig. 1A). Large LDs are probably the result of individual LD growth or the fusion of smaller LDs. After fertilization, the early embryos utilize pyruvate and lactate as their primary energy source for development, whereas the requirement for glucose increases from the morula stage on (i.e. glucose metabolism increases rapidly) (Leese \& Barton 1984, Leese 2012). This dramatic change in nutritional demand (energy metabolism) likely also affects the LD morphology. In particular, much energy is required for blastocoel formation and hatching. Thus, it follows that LDs enlarge in preparation to store additional neutral lipids, which will be required once energy consumption rises. Larger LDs also allow for increased contact area with other organelles, thereby facilitating lipid exchange and replenishment. However, the molecular mechanism of LD enlargement remains largely unknown. It is possible

\section{Box 2. Delipidation of mouse MII oocytes}

A method based on two-step centrifugation combined with hyperosmotic treatment was recently developed to remove LDs from mouse MII oocytes (Aizawa et al. 2019). MII oocytes are centrifuged at low speed (4200 g, $10 \mathrm{~min}$ ) to aggregate the LDs close to the cell membrane. Subsequently, the oocytes are centrifuged at high speed (9500 g, $10 \mathrm{~min}$ ) under hyperosmolar conditions (approximately 440 mOsm) to release the aggregated LDs out to the perivitelline space (PV; the gap between the cell membrane and the zona pellucida), where they can be removed by glass capillary tubes. The majority of MII oocytes subjected to this treatment survive. However, this method cannot remove LDs from cleavage-stage embryos. This is probably because LDs disperse and increase their physical interactions with other organelles after fertilization, making it more difficult for LDs to be released into the PV. The ease with which LDs can be removed from MII oocytes depends on the mouse strain. Mice with more clustered LDs in their MII oocytes (e.g. C57BL/6 and C3H mice) more efficiently release their LDs into the $\mathrm{PV}$, whereas those with more dispersed LDs (e.g. BALB/c and DBA/2 mice) are less efficient at releasing their LDs. The release efficiency correlates with the LD content of MII oocytes; therefore, when the LD content of MII oocytes is increased due to (short-term) HFD intake, the release efficiency increases (Aizawa et al. 2021). 
that similar MCS-mediated interactions among LDs, as discussed previously, may be involved in the clustering and enlargement of LDs.

\section{Differences in LD morphology among mouse strains}

The morphology of LDs in mouse oocytes varies among strains (Fig. 2). Based on the morphology of LDs in MII oocytes, strains can be classified according to those that are likely to form clusters (e.g. C57BL/6 and $\mathrm{C} 3 \mathrm{H}$ mice) and those that are likely to be dispersed (e.g. BALB/C and DBA/2 mice) (Fig. 2). What causes these differences in LDs between strains? One possibility is the difference in types and expression levels of proteins on the LD surface among strains; mice that are likely to have clustered LDs may express proteins that promote contact between LDs. Another possibility is that differences in the molecular species of phospholipids that make up the phospholipid monolayer of LDs (physical property of the LD surface) affect the morphology and size of LDs. The majority of phospholipids on the LD surface in mammalian cells are phosphatidylcholine (PC; $50-60 \%$ ) or phosphatidylethanolamine (PE; 20-30\%) (TauchiSato et al. 2002). PC is cylindrical and contributes to the stability of the phospholipid monolayer's planar structure, allowing for LDs with larger surface area. In contrast, conical phospholipids such as PE facilitate the formation of a negative curvature in the monolayer, which destabilizes the LDs, causing them to enlarge by fusing with other LDs (see the review by Thiam et al. 2013). Even though the LD morphology in early cleavage-stage embryos varies among mouse strains, LD enlargement after the morula stage seems to be common to all mouse strains. The physical properties of the LD surface during early embryonic development may differ among mouse strains, thereby resulting in a variety of LD morphologies. After the morula stage, these physical properties may become similar, and this may be the reason why large LDs are commonly observed in all mouse strains.

\section{The role of LDs in embryonic diapause}

ED is a temporary and reversible cessation of embryonic development at the blastocyst stage and is characterized by delayed implantation in the uterus. ED is observed in over 130 mammalian species (Fenelon et al. 2014, Renfree \& Fenelon 2017). The length of ED varies greatly depending on the species and can last from a few days to several months (e.g. 5 months in the roe deer, Capreolus capreolus; Aitken 1981). Once the uterine environment becomes favorable, the blastocyst resumes development and implants normally. ED is considered a reproductive strategy for ensuring the survival of neonates and is associated with metabolic stress, lactation period, and temperature change (Fenelon et al. 2014). During ED, blastocysts have active lipolysis and decreased TG content (Hussein et al. 2020). ED also involves AMPK and mechanistic target of rapamycin signaling (Lee et al. 2011, Bulut-Karslioglu et al. 2016, Hussein et al. 2020), both of which regulate the cellular energy balance as well as autophagy. This suggests that ED is maintained by complex molecular mechanisms in response to metabolic changes (e.g. amino acid concentration and composition of the uterine fluid).

More recently, Arena et al. reported that LDs are the main energy source for maintaining ED and that there is a positive correlation between the LD content in the oocyte and the duration of diapause (Arena et al. 2021). This means that animals such as mustelids with a higher LD content in their oocytes have a longer period of ED. However, given that the authors removed the LDs from one-cell embryos, the blastocysts in their mouse study might have accumulated a certain amount of newly synthesized LDs in the 4 days leading up to the blastocyst stage (this is because mouse oocytes and early cleavage-stage embryos have a mechanism for maintaining a certain amount of LDs (Aizawa et al. 2019), as will be explained later). Because the morphology and size of LDs change dynamically during preimplantation embryonic development, it is also possible that the enlarged LDs in the post-morula stage are responsible for supplying the energy required for ED. In either case, further studies are necessary to clarify whether the amount of LDs in a mature oocyte plays a role in determining the length of ED.

\section{Factors influencing the amount of LDs in mammalian oocytes: obesity and high-fat diet intake}

It is well known that maternal obesity has a major impact on oocyte competence. Meiotic errors during oogenesis, mitochondrial dysfunction, genetic and epigenetic instability, and embryonic development failure are typical consequences of maternal obesity (Igosheva et al. 2010, Luzzo et al. 2012, Ge et al. 2014, Wu et al. 2015, Zhang et al. 2015, Han et al. 2018). In addition, the LD content of mouse oocytes increases with obesity ( $\mathrm{Wu}$ et al. 2010). Many of these studies were performed using mice with long-term high-fat diet (HFD)-induced obesity, but our recent study revealed that LD content also increases in MII oocytes after short-term (a few days) HFD intake (not accompanied by obesity) (Aizawa et al. 2021). Mass spectrometric analysis confirmed that short-term HFD intake increases the levels of all TG species in MIl oocytes, suggesting that even short-term HFD intake would rapidly affect the LD content of mature oocytes. How does this occur? The fact that only a few days of HFD intake is sufficient to increase the LD content of ovulated oocytes suggests that fully grown oocytes in antral follicles are most susceptible to the effect of HFD intake. Because ovarian 
follicles at this stage are filled with follicular fluid, HFD intake is expected to increase the concentration of fatty acids in the follicular fluid, which would be taken up by the oocyte and/or stored in the LDs as neutral lipids. Furthermore, an oocyte growing within a follicle is surrounded by granulosa and cumulus cells, so the fatty acids in the follicular fluid may first be taken up by these cells and then transported to the oocyte via gap junctions (Carabatsos et al. 2000) or transzonal projections (TZPs) (Albertini et al. 2001). It was also reported that fatty acid binding protein 3 (FABP3) is involved in the transfer of fatty acids from cumulus cells to the oocytes via TZPs and that increased expression level of FABP3 correlates with increased LD content in bovine oocytes during in vitro maturation (Del Collado et al. 2017). Additionally, because short-term intake of HFD did not affect oocyte competence (i.e. oocytes from HFD-fed females were able to be fertilized and then developed normally; R Aizawa \& S Tsukamoto, unpublished observations), it is possible that the more toxic fatty acids (i.e. saturated fatty acids such as palmitic acid) that increased in follicular fluid are actively taken up by the cumulus cells and converted to less toxic fatty acids (Aardema et al. 2013, 2018), thereby protecting the oocyte from lipotoxicity. Because interaction between follicular somatic cells and the oocytes is essential for follicle growth (see the reviews by Eppig 2001, Matzuk et al. 2002), the amount of LDs in an oocyte may be regulated in cooperation with the surrounding cells. Taken together, these findings suggest that the amount of LDs stored in a growing oocyte inside the follicle is regulated through bidirectional communication between the oocytes and the surrounding somatic cells in response to changes in the follicular environment.

Meanwhile, the concentration of fatty acids and the amount of neutral lipids in the follicular fluid increase with BMI in humans (Robker et al. 2009, Valckx et al. 2012, Dunning et al. 2014, Pantasri et al. 2015). These concentrations could serve as indicators of the lipid metabolism status (i.e. quality of lipids) of the mature oocyte in the follicle. However, it is important to bear in mind that the concentration of fatty acids and the amount of neutral lipids in the follicular fluid might change according to the types and quantities of dietderived lipids within the short term (a few days) even in non-obese individuals (BMI within a normal range).

\section{Homeostatic regulation of LD content in oocytes and early embryos}

Removal of LDs from mouse MII oocytes by two-step centrifugation (delipidation, Box 2) results in rapid synthesis of new LDs, whether or not fatty acids are present in the culture medium. In contrast, transplantation of LDs isolated from MII oocytes into the cytoplasm of fertilized embryos (which leads to abnormal LD accumulation) results in the rapid breakdown of the accumulated LDs (Aizawa et al. 2019). These findings indicate that some mechanism exists in mouse oocytes and early cleavage-stage embryos that regulates the balance of LD synthesis and LD degradation in order to maintain a certain amount of LDs. In other words, the LD content of mammalian oocytes and embryos is considered to be homeostatically regulated. This means that a deficiency or excess of LDs would be rapidly corrected to restore homeostasis. Furthermore, failure to maintain LD homeostasis (i.e. LD biogenesis and degradation are thrown off balance) would reduce the rates of successful oocyte maturation and preimplantation embryonic development (Fig. 3).

What mechanism maintains LD homeostasis? There are several pathways for the synthesis of LDs, but here we focus on the synthesis of neutral lipids from fatty acids. Fatty acids are converted to their active form (acyl-CoA) by intracellular acyl-CoA synthetases. There are several different types of acyl-CoA synthetases. The long-chain acyl-CoA synthetase (ACSL) family members (ACSL1, $3,4,5$, and 6) convert long-chain fatty acids (12-20

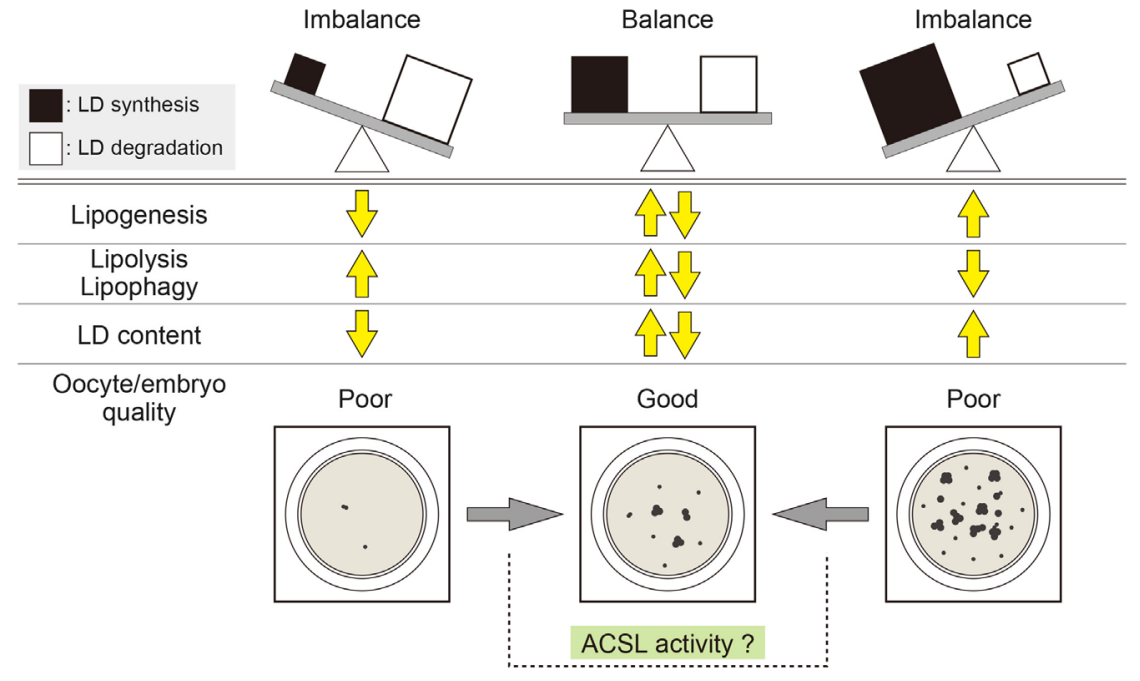

Figure 3 Model illustrating the homeostatic regulation of balance between lipogenesis and lipolysis/lipophagy in mammalian oocytes and early embryos. The subtle balance between lipogenesis (LD synthesis) and lipolysis/ lipophagy (LD degradation) is critical for optimizing LD content in mammalian oocytes and early embryos. Imbalance (depletion or overabundance) in LD content can negatively affect not only lipid metabolism but also glucose homeostasis and mitochondria metabolism, leading to reduce oocyte/embryo developmental competence (viability). To maintain the proper amount of LDs in the cells, lipogenesis and lipolysis/lipophagy are cooperatively and complementarily regulated, most likely through ACSL activity (predominantly ACSL3). 
carbon atoms) into their respective acyl-CoA molecules, which are then utilized for the synthesis of neutral lipids. ACSL proteins are involved in lipid metabolism, and ACSL3 interacts with LDs (Brasaemle et al. 2004, Fujimoto et al. 2004, Kassan et al. 2013). In mature mouse oocytes, ACSL3 is the most highly expressed among ACSL family proteins (Aizawa et al. 2019). Culturing oocytes and early embryos with an ACSLspecific inhibitor (Triacsin C; Tomoda et al. 1987) not only results in complete suppression of new LD synthesis but also in the degradation of LDs that were already present in the cells. Furthermore, the development of early embryos lacking LDs due to Triacsin C treatment is arrested at the eight-cell stage (Aizawa et al. 2019). These findings indicate that ACSL3 is a key player for LD homeostasis and that the dysfunction of this protein causes embryonic lethality (see also Fig. 3). It is unclear whether homeostatic regulation of LDs occurs via ACSL3 in porcine and bovine embryos with larger amounts of LDs. However, given findings that (1) Acsl3 mRNA is highly expressed in bovine embryos at the morula stage, when LD levels are highest (Sudano et al. 2016), and (2) the expression of genes related to TG synthesis, including GPAT1 and DGAT, as well as LIPIN1, a critical regulator of lipid metabolism, is increased after delipidation in porcine embryos (Zeng et al. 2017), it is possible that the mechanism of $L D$ regulation is conserved across mammalian species.

\section{Concluding remarks}

Many previous studies on the role of LDs during oocyte maturation and preimplantation embryonic development have focused on porcine and bovine oocytes/embryos, which contain high levels of LDs. More recent studies have revealed the importance of maintaining a certain level of LDs in the cytoplasm and have uncovered a correlation between the amount of LDs in mammalian oocytes and the length of ED. However, the critical mysteries of how the amount of LDs to be stored in the oocyte is determined and how LD homeostasis is maintained remain unanswered. It is also not known why porcine and bovine oocytes are able to retain large quantities of LDs while mouse and human oocytes are not.

The content and morphology of LDs in different mammalian species and even different mouse strains are likely determined by (1) the proteins expressed on the LD surface and (2) the physical properties of the LD phospholipid monolayer. Considering that the proteins expressed on the LD surface are involved in the clustering and fusion (enlargement) of LDs in addition to preventing their degradation by lipases, LDs in porcine and bovine oocytes/embryos with higher levels of LDs are likely covered by such proteins. In contrast, we would expect the LDs in mouse and human oocytes/embryos with lower levels of LDs to express either no such (functional) proteins, or if they do, to be easily degradable (transiently expressed). Moreover, it is possible that differences in the physical properties of the LD phospholipid monolayer among mammalian species might affect the quantity and morphology of their respective LDs. The phospholipid composition of LDs and LD-related proteins might be affected by obesity and HFD intake, leading to fluctuations in LD content. In mammalian oocytes and early embryos, LDs are thought to be degraded by lipolysis for energy production and subjected to lipophagy to maintain their quality. The latter is thought to play an important role in the maintenance of cellular homeostasis in porcine and bovine oocytes/embryos, which contain large amounts of LDs. In either case, the molecular mechanism of this homeostasis remains to be elucidated.

It is expected that the factors determining LD content and their regulatory mechanism as well as the molecular mechanism for regulating the quality of LDs in mammalian oocytes and preimplantation embryos will be elucidated by proteome and lipidome analyses in the near future.

\section{Declaration of interest}

The authors declare that there is no conflict of interest that could be perceived as prejudicing the impartiality of this review.

\section{Funding}

This work was supported by the Grant-in-Aid for Scientific Research on Innovative Areas (JSPS KAKENHI Grant No. $21 \mathrm{H} 00240)$ and the Takeda Science Foundation (visionary research) to $\mathrm{S} \mathrm{T}$.

\section{Author contribution statement}

M I and S T surveyed the literature and produced the figures. $\mathrm{R}$ A and S T performed the experiments. S T conceived the study and wrote the manuscript with support from M I, R A, and J M. All authors discussed the results and contributed to the final manuscript.

\section{References}

Aardema $\mathrm{H}$, Lolicato $\mathrm{F}$, van de Lest $\mathrm{CH}$, Brouwers JF, Vaandrager $\mathrm{AB}$, van Tol HT, Roelen BA, Vos PL, Helms JB \& Gadella BM 2013 Bovine cumulus cells protect maturing oocytes from increased fatty acid levels by massive intracellular lipid storage. Biology of Reproduction 88164. (https://doi.org/10.1095/biolreprod.112.106062)

Aardema H, Vos PLAM \& Gadella BM 2018 Cumulus cells protect the oocyte against saturated free fatty acids. Animal Reproduction 15 737-750. (https://doi.org/10.21451/1984-3143-AR2018-0063)

Aitken RJ 1981 Aspects of delayed implantation in the roe deer (Capreolus capreolus). Journal of Reproduction and Fertility: Supplement 29 83-95.

Aizawa R, Ibayashi M, Tatsumi T, Yamamoto A, Kokubo T, Miyasaka N, Sato K, Ikeda S, Minami N \& Tsukamoto S 2019 Synthesis and maintenance of lipid droplets are essential for mouse preimplantation embryonic development. Development 146 dev181925. (https://doi. org/10.1242/dev.181925) 
Aizawa R, Ibayashi M, Hatakeyama T, Tatsumi T \& Tsukamoto S 2021 Impact of short-term high-fat feeding on lipid droplet content in mouse oocytes. Journal of Reproduction and Development 67 73-77. (https:// doi.org/10.1262/jrd.2020-094)

Albertini DF, Combelles CM, Benecchi E \& Carabatsos MJ 2001 Cellular basis for paracrine regulation of ovarian follicle development. Reproduction 121 647-653. (https://doi.org/10.1530/rep.0.1210647)

Arena R, Bisogno S, Gasior $\mathrm{E}$, Rudnicka J, Bernhardt L, Haaf T, Zacchini $\mathrm{F}_{\text {, }}$ Bochenek M, Fic K, Bik E et al. 2021 Lipid droplets in mammalian eggs are utilized during embryonic diapause. PNAS 118 e2018362118. (https://doi.org/10.1073/pnas.2018362118)

Benador IY, Veliova M, Mahdaviani K, Petcherski A, Wikstrom JD, Assali EA Acin-Perez R, Shum M, Oliveira MF, Cinti S et al. 2018 Mitochondria bound to lipid droplets have unique bioenergetics, composition, and dynamics that support lipid droplet expansion. Cell Metabolism 27869 885.e6. (https://doi.org/10.1016/j.cmet.2018.03.003)

Bersuker K, Peterson CWH, To M, Sahl SJ, Savikhin V, Grossman EA, Nomura DK \& Olzmann JA 2018 A proximity labeling strategy provides insights into the composition and dynamics of lipid droplet proteomes. Developmental Cell 44 97.e7-112.e7. (https://doi.org/10.1016/j. devcel.2017.11.020)

Bickel PE, Tansey JT \& Welte MA 2009 PAT proteins, an ancient family of lipid droplet proteins that regulate cellular lipid stores. Biochimica et Biophysica Acta 1791 419-440. (https://doi.org/10.1016/j. bbalip.2009.04.002)

Bosch M, Sanchez-Alvarez M, Fajardo A, Kapetanovic R, Steiner B, Dutra F, Moreira L, Lopez JA, Campo R, Mari M et al. 2020 Mammalian lipid droplets are innate immune hubs integrating cell metabolism and host defense. Science 370 aay8085. (https://doi.org/10.1126/science. aay8085)

Bradley J \& Swann K2019 Mitochondria and lipid metabolism in mammalian oocytes and early embryos. International Journal of Developmental Biology 63 93-103. (https://doi.org/10.1387/ijdb.180355ks)

Bradley J, Pope I, Masia F, Sanusi R, Langbein W, Swann K \& Borri P 2016 Quantitative imaging of lipids in live mouse oocytes and early embryos using CARS microscopy. Development 143 2238-2247. (https://doi. org/10.1242/dev.129908)

Bradley J, Pope I, Wang Y, Langbein W, Borri P \& Swann K 2019 Dynamic label-free imaging of lipid droplets and their link to fatty acid and pyruvate oxidation in mouse eggs. Journal of Cell Science 132 jcs228999. (https:// doi.org/10.1242/jcs.228999)

Brasaemle DL, Barber T, Wolins NE, Serrero G, Blanchette-Mackie EJ \& Londos C 1997 Adipose differentiation-related protein is an ubiquitously expressed lipid storage droplet-associated protein. Journal of Lipid Research 38 2249-2263. (https://doi.org/10.1016/S00222275(20)34939-7)

Brasaemle DL, Dolios G, Shapiro L \& Wang R 2004 Proteomic analysis of proteins associated with lipid droplets of basal and lipolytically stimulated 3T3-L1 adipocytes. Journal of Biological Chemistry 279 46835-46842. (https://doi.org/10.1074/jbc.M409340200)

Buhman KK, Chen HC \& Farese Jr RV 2001 The enzymes of neutral lipid synthesis. Journal of Biological Chemistry 276 40369-40372. (https:// doi.org/10.1074/jbc.R100050200)

Bulut-Karslioglu A, Biechele S, Jin H, Macrae TA, Hejna M, Gertsenstein M, Song JS \& Ramalho-Santos M 2016 Inhibition of mTOR induces a paused pluripotent state. Nature $\mathbf{5 4 0}$ 119-123. (https://doi.org/10.1038/ nature20578)

Carabatsos MJ, Sellitto C, Goodenough DA \& Albertini DF 2000 Oocyte-granulosa cell heterologous gap junctions are required for the coordination of nuclear and cytoplasmic meiotic competence. Developmental Biology 226 167-179. (https://doi.org/10.1006/ dbio.2000.9863)

Chang BH, Li L, Paul A, Taniguchi S, Nannegari V, Heird WC \& Chan L 2006 Protection against fatty liver but normal adipogenesis in mice lacking adipose differentiation-related protein. Molecular and Cellular Biology 26 1063-1076. (https://doi.org/10.1128/MCB.26.3.10631076.2006)

Del Collado M, da Silveira JC, Sangalli JR, Andrade GM, Sousa LRDS, Silva LA, Meirelles FV \& Perecin F 2017 Fatty acid binding protein 3 and transzonal projections are involved in lipid accumulation during in vitro maturation of bovine oocytes. Scientific Reports 7 2645. (https:// doi.org/10.1038/s41598-017-02467-9)
Downs SM, Mosey JL \& Klinger J 2009 Fatty acid oxidation and meiotic resumption in mouse oocytes. Molecular Reproduction and Development 76 844-853. (https://doi.org/10.1002/mrd.21047)

Dunning KR \& Robker RL 2012 Promoting lipid utilization with l-carnitine to improve oocyte quality. Animal Reproduction Science 134 69-75. (https://doi.org/10.1016/j.anireprosci.2012.08.013)

Dunning KR, Cashman K, Russell DL, Thompson JG, Norman RJ \& Robker RL 2010 Beta-oxidation is essential for mouse oocyte developmental competence and early embryo development. Biology of Reproduction 83 909-918. (https://doi.org/10.1095/biolreprod.110.084145)

Dunning KR, Russell DL \& Robker RL 2014 Lipids and oocyte developmental competence: the role of fatty acids and beta-oxidation. Reproduction 148 R15-R27. (https://doi.org/10.1530/REP-13-0251)

Eppig JJ 2001 Oocyte control of ovarian follicular development and function in mammals. Reproduction 122 829-838. (https://doi. org/10.1530/rep.0.1220829)

Fenelon JC, Banerjee A \& Murphy BD 2014 Embryonic diapause: development on hold. International Journal of Developmental Biology 58 163-174. (https://doi.org/10.1387/ijdb.140074bm)

Fujimoto Y, Itabe H, Sakai J, Makita M, Noda J, Mori M, Higashi Y, Kojima S \& Takano T 2004 Identification of major proteins in the lipid dropletenriched fraction isolated from the human hepatocyte cell line HuH7. Biochimica et Biophysica Acta 1644 47-59. (https://doi.org/10.1016/j. bbamcr.2003.10.018)

Ge ZJ, Luo SM, Lin F, Liang QX, Huang L, Wei YC, Hou Y, Han ZM, Schatten H \& Sun QY 2014 DNA methylation in oocytes and liver of female mice and their offspring: effects of high-fat-diet-induced obesity. Environmental Health Perspectives 122 159-164. (https://doi. org/10.1289/ehp.1307047)

Greenberg AS, Egan JJ, Wek SA, Moos Jr MC, Londos C \& Kimmel AR 1993 Isolation of CDNAs for perilipins A and B: sequence and expression of lipid droplet-associated proteins of adipocytes. PNAS 90 12035-12039. (https://doi.org/10.1073/pnas.90.24.12035)

Han L, Ren C, Li L, Li X, Ge J, Wang H, Miao YL, Guo X, Moley KH, Shu W et al. 2018 Embryonic defects induced by maternal obesity in mice derive from Stella insufficiency in oocytes. Nature Genetics $\mathbf{5 0}$ 432-442. (https://doi.org/10.1038/s41588-018-0055-6)

Herms A, Bosch M, Ariotti N, Reddy BJ, Fajardo A, Fernandez-Vidal A, Alvarez-Guaita A, Fernandez-Rojo MA, Rentero C, Tebar F et al. 2013 Cell-to-cell heterogeneity in lipid droplets suggests a mechanism to reduce lipotoxicity. Current Biology 23 1489-1496. (https://doi. org/10.1016/j.cub.2013.06.032)

Herms A, Bosch M, Reddy BJ, Schieber NL, Fajardo A, Ruperez C, Fernandez-Vidal A, Ferguson C, Rentero C, Tebar F et al. 2015 AMPK activation promotes lipid droplet dispersion on detyrosinated microtubules to increase mitochondrial fatty acid oxidation. Nature Communications 6 7176. (https://doi.org/10.1038/ncomms8176)

Hodges BD \& Wu CC 2010 Proteomic insights into an expanded cellular role for cytoplasmic lipid droplets. Journal of Lipid Research $\mathbf{5 1}$ 262-273. (https://doi.org/10.1194/jlr.R003582)

Hussein AM, Wang Y, Mathieu J, Margaretha L, Song C, Jones DC, Cavanaugh C, Miklas JW, Mahen E, Showalter MR et al. 2020 Metabolic control over mTOR-dependent diapause-like state. Developmental Cell 52 236.e7-250.e7. (https://doi.org/10.1016/j.devcel.2019.12.018)

Ichimura Y, Kumanomidou T, Sou YS, Mizushima T, Ezaki J, Ueno T, Kominami E, Yamane T, Tanaka K \& Komatsu M 2008 Structural basis for sorting mechanism of p62 in selective autophagy. Journal of Biological Chemistry 283 22847-22857. (https://doi.org/10.1074/jbc. M802182200)

Igosheva N, Abramov AY, Poston L, Eckert JJ, Fleming TP, Duchen MR \& McConnell J 2010 Maternal diet-induced obesity alters mitochondrial activity and redox status in mouse oocytes and zygotes. PLOS ONE 5 e10074. (https://doi.org/10.1371/journal.pone.0010074)

loannou MS, Jackson J, Sheu SH, Chang CL, Weigel AV, Liu H, Pasolli HA, Xu CS, Pang S, Matthies D et al. 2019 Neuron-astrocyte metabolic coupling protects against activity-induced fatty acid toxicity. Cell $\mathbf{1 7 7}$ 1522.e14-1535.e14. (https://doi.org/10.1016/j.cell.2019.04.001)

Kassan A, Herms A, Fernandez-Vidal A, Bosch M, Schieber NL, Reddy BJ, Fajardo A, Gelabert-Baldrich M, Tebar F, Enrich C et al. 2013 Acyl-CoA synthetase 3 promotes lipid droplet biogenesis in ER microdomains. Journal of Cell Biology 203 985-1001. (https://doi.org/10.1083/ jcb.201305142) 
Kikuchi K, Ekwall H, Tienthai P, Kawai Y, Noguchi J, Kaneko H \& RodriguezMartinez H 2002 Morphological features of lipid droplet transition during porcine oocyte fertilisation and early embryonic development to blastocyst in vivo and in vitro. Zygote 10 355-366. (https://doi. org/10.1017/s0967199402004100)

Laufman O, Perrino J \& Andino R 2019 Viral generated inter-organelle contacts redirect lipid flux for genome replication. Cell 178 275.e16289.e16. (https://doi.org/10.1016/j.cell.2019.05.030)

Lee JE, Oh HA, Song H, Jun JH, Roh CR, Xie H, Dey SK \& Lim HJ 2011 Autophagy regulates embryonic survival during delayed implantation. Endocrinology 152 2067-2075. (https://doi.org/10.1210/en.2010-1456)

Leese HJ 2012 Metabolism of the preimplantation embryo: 40 years on. Reproduction 143 417-427. (https://doi.org/10.1530/REP-11-0484)

Leese HJ \& Barton AM 1984 Pyruvate and glucose uptake by mouse ova and preimplantation embryos. Journal of Reproduction and Fertility $\mathbf{7 2}$ 9-13. (https://doi.org/10.1530/jrf.0.0720009)

Li Z, Thiel K, Thul PJ, Beller M, Kuhnlein RP \& Welte MA 2012 Lipid droplets control the maternal histone supply of Drosophila embryos. Current Biology 22 2104-2113. (https://doi.org/10.1016/j.cub.2012.09.018)

Luzzo KM, Wang Q, Purcell SH, Chi M, Jimenez PT, Grindler N, Schedl T \& Moley KH 2012 High fat diet induced developmental defects in the mouse: oocyte meiotic aneuploidy and fetal growth retardation/brain defects. PLOS ONE 7 e49217. (https://doi.org/10.1371/journal.pone.0049217)

Martinez-Botas J, Anderson JB, Tessier D, Lapillonne A, Chang BH, Quast MJ, Gorenstein D, Chen KH \& Chan L 2000 Absence of perilipin results in leanness and reverses obesity in $\operatorname{Lepr}(\mathrm{db} / \mathrm{db})$ mice. Nature Genetics 26 474-479. (https://doi.org/10.1038/82630)

Matzuk MM, Burns KH, Viveiros MM \& Eppig JJ 2002 Intercellular communication in the mammalian ovary: oocytes carry the conversation. Science 296 2178-2180. (https://doi.org/10.1126/science.1071965)

McKeegan PJ \& Sturmey RG 2011 The role of fatty acids in oocyte and early embryo development. Reproduction, Fertility, and Development $\mathbf{2 4}$ 59-67. (https://doi.org/10.1071/RD11907)

McManaman JL, Bales ES, Orlicky DJ, Jackman M, MacLean PS, Cain S, Crunk AE, Mansur A, Graham CE, Bowman TA et al. 2013 Perilipin-2-null mice are protected against diet-induced obesity, adipose inflammation, and fatty liver disease. Journal of Lipid Research 54 1346-1359. (https:// doi.org/10.1194/jIr.M035063)

Mejhert N, Kuruvilla L, Gabriel KR, Elliott SD, Guie MA, Wang H, Lai ZW, Lane EA, Christiano R, Danial NN et al. 2020 Partitioning of MLXfamily transcription factors to lipid droplets regulates metabolic gene expression. Molecular Cell 77 1251-1264. (https://doi.org/10.1016/j. molcel.2020.01.014)

Mizushima N 2007 Autophagy: process and function. Genes and Development 21 2861-2873. (https://doi.org/10.1101/gad.1599207)

Nagashima H, Kashiwazaki N, Ashman RJ, Grupen CG \& Nottle MB 1995 Cryopreservation of porcine embryos. Nature 374 416. (https://doi. org/10.1038/374416a0)

Nguyen TB, Louie SM, Daniele JR, Tran Q, Dillin A, Zoncu R, Nomura DK \& Olzmann JA 2017 DGAT1-dependent lipid droplet biogenesis protects mitochondrial function during starvation-induced autophagy. Developmental Cell 42 9-21. (https://doi.org/10.1016/j. devcel.2017.06.003)

Ohsaki Y, Kawai T, Yoshikawa Y, Cheng J, Jokitalo E \& Fujimoto T 2016 PML isoform II plays a critical role in nuclear lipid droplet formation. Journal of Cell Biology 212 29-38. (https://doi.org/10.1083/ jcb.201507122)

Pantasri T, Wu LL, Hull ML, Sullivan TR, Barry M, Norman RJ \& Robker RL 2015 Distinct localisation of lipids in the ovarian follicular environment. Reproduction, Fertility, and Development 27 593-601. (https://doi. org/10.1071/RD14321)

Prinz WA 2014 Bridging the gap: membrane contact sites in signaling, metabolism, and organelle dynamics. Journal of Cell Biology 205 759-769. (https://doi.org/10.1083/jcb.201401126)

Rambold AS, Cohen S \& Lippincott-Schwartz J 2015 Fatty acid trafficking in starved cells: regulation by lipid droplet lipolysis, autophagy, and mitochondrial fusion dynamics. Developmental Cell 32 678-692. (https://doi.org/10.1016/j.devcel.2015.01.029)

Renfree MB \& Fenelon JC 2017 The enigma of embryonic diapause. Development 144 3199-3210. (https://doi.org/10.1242/dev.148213)

Robker RL, Akison LK, Bennett BD, Thrupp PN, Chura LR, Russell DL, Lane M \& Norman RJ 2009 Obese women exhibit differences in ovarian metabolites, hormones, and gene expression compared with moderateweight women. Journal of Clinical Endocrinology and Metabolism 94 1533-1540. (https://doi.org/10.1210/jc.2008-2648)

Romanauska A \& Kohler A 2018 The inner nuclear membrane is a metabolically active territory that generates nuclear lipid droplets. Cell 174 700-715. (https://doi.org/10.1016/j.cell.2018.05.047)

Sastre D, da Costa NN, de Sa AL, Conceicao SD, Chiaratti MR, Adona PR, Guemra S, Meirelles FV, Santos Sdo Sdo S, Sena L et al. 2014 Expression of PLIN2 and PLIN3 during oocyte maturation and early embryo development in cattle. Theriogenology 81 326-331. (https://doi. org/10.1016/j.theriogenology.2013.10.002)

Schott MB, Weller SG, Schulze RJ, Krueger EW, Drizyte-Miller K, Casey CA \& McNiven MA 2019 Lipid droplet size directs lipolysis and lipophagy catabolism in hepatocytes. Journal of Cell Biology 218 3320-3335. (https://doi.org/10.1083/jcb.201803153)

Servetnick DA, Brasaemle DL, Gruia-Gray J, Kimmel AR, Wolff J \& Londos C 1995 Perilipins are associated with cholesteryl ester droplets in steroidogenic adrenal cortical and Leydig cells. Journal of Biological Chemistry 270 16970-16973. (https://doi.org/10.1074/ jbc.270.28.16970)

Soltysik K, Ohsaki Y, Tatematsu T, Cheng J, Maeda A, Morita SY \& Fujimoto T 2021 Nuclear lipid droplets form in the inner nuclear membrane in a seipin-independent manner. Journal of Cell Biology 220 e202005026. (https://doi.org/10.1083/jcb.202005026)

Sturmey RG, O'Toole PJ \& Leese HJ 2006 Fluorescence resonance energy transfer analysis of mitochondrial:lipid association in the porcine oocyte. Reproduction 132 829-837. (https://doi.org/10.1530/REP-06-0073)

Sturmey RG, Reis A, Leese HJ \& McEvoy TG 2009 Role of fatty acids in energy provision during oocyte maturation and early embryo development. Reproduction in Domestic Animals 44 (Supplement 3) 50-58. (https://doi.org/10.1111/j.1439-0531.2009.01402.x)

Sudano MJ, Rascado TD, Tata A, Belaz KR, Santos VG, Valente RS, Mesquita FS, Ferreira CR, Araujo JP, Eberlin MN et al. 2016 Lipidome signatures in early bovine embryo development. Theriogenology $\mathbf{8 6}$ 472-484. (https://doi.org/10.1016/j.theriogenology.2016.03.025)

Tansey JT, Sztalryd C, Gruia-Gray J, Roush DL, Zee JV, Gavrilova O, Reitman ML, Deng CX, Li C, Kimmel AR et al. 2001 Perilipin ablation results in a lean mouse with aberrant adipocyte lipolysis, enhanced leptin production, and resistance to diet-induced obesity. PNAS 98 6494-6499. (https://doi.org/10.1073/pnas.101042998)

Tatsumi T, Takayama K, Ishii S, Yamamoto A, Hara T, Minami N, Miyasaka N, Kubota T, Matsuura A, Itakura E et al. 2018 Forced lipophagy reveals that lipid droplets are required for early embryonic development in mouse. Development 145 dev161893. (https://doi.org/10.1242/dev.161893)

Tauchi-Sato K, Ozeki S, Houjou T, Taguchi R \& Fujimoto T 2002 The surface of lipid droplets is a phospholipid monolayer with a unique fatty acid composition. Journal of Biological Chemistry 277 44507-44512. (https://doi.org/10.1074/jbc.M207712200)

Thiam AR \& Dugail I 2019 Lipid droplet-membrane contact sites: from protein binding to function. Journal of Cell Science 132 jcs230169. (https://doi.org/10.1242/jcs.230169)

Thiam AR, Farese Jr RV \& Walther TC 2013 The biophysics and cell biology of lipid droplets. Nature Reviews: Molecular Cell Biology 14 775-786. (https://doi.org/10.1038/nrm3699)

Tomoda H, Igarashi K \& Omura S 1987 Inhibition of acyl-CoA synthetase by triacsins. Biochimica et Biophysica Acta 921 595-598. (https://doi. org/10.1016/0005-2760(87)90088-9)

Valckx SD, De Pauw I, De Neubourg D, Inion I, Berth M, Fransen E, Bols PE \& Leroy JL 2012 BMI-related metabolic composition of the follicular fluid of women undergoing assisted reproductive treatment and the consequences for oocyte and embryo quality. Human Reproduction $\mathbf{2 7}$ 3531-3539. (https://doi.org/10.1093/humrep/des350)

Valm AM, Cohen S, Legant WR, Melunis J, Hershberg U, Wait E, Cohen AR, Davidson MW, Betzig E \& Lippincott-Schwartz J 2017 Applying systemslevel spectral imaging and analysis to reveal the organelle interactome. Nature 546 162-167. (https://doi.org/10.1038/nature22369)

Valsangkar D \& Downs SM 2013 A requirement for fatty acid oxidation in the hormone-induced meiotic maturation of mouse oocytes. Biology of Reproduction 89 43. (https://doi.org/10.1095/biolreprod.113.109058)

Walther TC, Chung J \& Farese Jr RV 2017 Lipid droplet biogenesis. Annual Review of Cell and Developmental Biology 33 491-510. (https://doi. org/10.1146/annurev-cellbio-100616-060608) 
Wang H, Sreenivasan U, Hu H, Saladino A, Polster BM, Lund LM, Gong DW, Stanley WC \& Sztalryd C 2011 Perilipin 5, a lipid dropletassociated protein, provides physical and metabolic linkage to mitochondria. Journal of Lipid Research 52 2159-2168. (https://doi. org/10.1194/jlr.M017939)

Watanabe T, Thayil A, Jesacher A, Grieve K, Debarre D, Wilson T, Booth M \& Srinivas S 2010 Characterisation of the dynamic behaviour of lipid droplets in the early mouse embryo using adaptive harmonic generation microscopy. BMC Cell Biology 11 38. (https://doi.org/10.1186/14712121-11-38)

Wilfling F, Wang H, Haas JT, Krahmer N, Gould TJ, Uchida A, Cheng JX, Graham M, Christiano R, Frohlich F et al. 2013 Triacylglycerol synthesis enzymes mediate lipid droplet growth by relocalizing from the ER to lipid droplets. Developmental Cell 24 384-399. (https://doi.org/10.1016/j. devcel.2013.01.013)

Wolins NE, Quaynor BK, Skinner JR, Schoenfish MJ, Tzekov A \& Bickel PE 2005 S3-12, adipophilin, and TIP47 package lipid in adipocytes. Journal of Biological Chemistry 280 19146-19155. (https://doi.org/10.1074/jbc. M500978200)

Wu LL, Dunning KR, Yang X, Russell DL, Lane M, Norman RJ \& Robker RL 2010 High-fat diet causes lipotoxicity responses in cumulus-oocyte complexes and decreased fertilization rates. Endocrinology 151 5438-5445. (https://doi.org/10.1210/en.2010-0551)

Wu LL, Russell DL, Wong SL, Chen M, Tsai TS, John JCSt, Norman RJ, Febbraio MA, Carroll J \& Robker RL 2015 Mitochondrial dysfunction in oocytes of obese mothers: transmission to offspring and reversal by pharmacological endoplasmic reticulum stress inhibitors. Development 142 681-691. (https://doi.org/10.1242/dev.114850)

Yamaguchi T, Matsushita S, Motojima K, Hirose F \& Osumi T 2006 MLDP, a novel PAT family protein localized to lipid droplets and enriched in the heart, is regulated by peroxisome proliferator-activated receptor alpha. Journal of Biological Chemistry 281 14232-14240. (https://doi. org/10.1074/jbc.M601682200)

Yang X, Dunning KR, Wu LL, Hickey TE, Norman RJ, Russell DL, Liang X \& Robker RL 2010 Identification of perilipin-2 as a lipid droplet protein regulated in oocytes during maturation. Reproduction, Fertility, and Development 22 1262-1271. (https://doi.org/10.1071/RD10091)

Zechner R, Madeo F \& Kratky D 2017 Cytosolic lipolysis and lipophagy: two sides of the same coin. Nature Reviews: Molecular Cell Biology 18 671-684. (https://doi.org/10.1038/nrm.2017.76)

Zeng Y, Wang C, Niu Y, Chi D, Xu M, Si L, Qu X \& Li J 2017 The influence of delipidation on triglyceride and LIPIN1 of porcine embryos derived from parthenogenetic activation. Reproduction in Domestic Animals 52 842-850. (https://doi.org/10.1111/rda.12987)

Zhang RN, Fu XW, Jia BY, Liu C, Cheng KR \& Zhu SE 2014 Expression of perilipin 2 (PLIN2) in porcine oocytes during maturation. Reproduction in Domestic Animals 49 875-880. (https://doi.org/10.1111/rda.12386)

Zhang L, Han L, Ma R, Hou X, Yu Y, Sun S, Xu Y, SchedI T, Moley KH \& Wang Q 2015 Sirt3 prevents maternal obesity-associated oxidative stress and meiotic defects in mouse oocytes. Cell Cycle 14 2959-2968. (https:// doi.org/10.1080/15384101.2015.1026517)

Received 7 June 2021

First decision 27 July 2021

Revised manuscript received 27 August 2021

Accepted 13 September 2021 